\title{
Brain-computer interface algorithm based on wavelet-phase stability analysis in motor imagery experiment
}

\author{
Muhamad Firdaus Mohd Rafi a , Arief Ruhullah A Harris ${ }^{b}$, Tan Tian Swee ${ }^{b^{*}}$, Leong Kah Meng ${ }^{a}$, \\ Tan Jia Hou ${ }^{a}$, Kelvin Ling Chia Hiik ${ }^{a}$, Tengku Ahmad Iskandar Tengku Alang a, Azli Yahya ${ }^{a}$, \\ Joyce Sia Sin Yin ${ }^{a}$, Matthias Tiong Foh Thye ${ }^{a}$, Sameen Ahmed Malik ${ }^{a}$ \\ a Department of Biomedical Engineering, School of Biomedical Engineering and Health Sciences (SKBSK), Faculty of Engineering, Universiti \\ Teknologi Malaysia, 81310 UTM Johor Bahru, Johor, Malaysia \\ ${ }^{b}$ Bioinspired Device and Tissue Engineering (BIOINSPIRA), School of Biomedical Engineering and Health Sciences, (SKBSK), Faculty of \\ Engineering, Universiti Teknologi Malaysia, 81310 UTM Johor Bahru, Johor, Malaysia
}

* Corresponding author: tantswee@biomedical.utm.my

\section{Article history}

Received 17 April 2018

Revised 19 August 2019

Accepted 14 September 2019

Published Online 15 April 2020

\begin{abstract}
Severe movement or motor disability diseases such as amyotrophic lateral sclerosis (ALS), cerebral palsy (CB), and muscular dystrophy (MD) are types of diseases which lead to the total of function loss of body parts, usually limbs. Patient with an extreme motor impairment might suffers a lockedin state, resulting in the difficulty to perform any physical movements. These diseases are commonly being treated by a specific rehabilitation procedure with prescribed medication. However, the recovery process is time-consuming through such treatments. To overcome these issues, BrainComputer Interface system is introduced in which one of its modalities is to translate thought via electroencephalography (EEG) signals by the user and generating desired output directly to an external artificial control device or human augmentation. Here, phase synchronization is implemented to complement the $\mathrm{BCl}$ system by analyzing the phase stability between two input signals. The motor imagery-based experiment involved ten healthy subjects aged from 24 to 30 years old with balanced numbers between male and female. Two aforementioned input signals are the respective reference data and the real time data were measured by using phase stability technique by indicating values range from 0 (least stable) to 1 (most stable). Prior to that, feature extraction was utilized by applying continuous wavelet transform (CWT) to quantify significant features on the basis of motor imagery experiment which are right and left imaginations. The technique was able to segregate different classes of motor imagery task based on classification accuracy. This study affirmed the approach's ability to achieve high accuracy output measurements.
\end{abstract}

Keywords: Brain-Computer Interface, electroencephalography, motor imagery, phase stability analysis, thresholding technique.

(C) 2020 Penerbit UTM Press. All rights reserved

\section{INTRODUCTION}

Physical disability is among the leading cases in Malaysia [1] related with many types of diseases such as amyotrophic lateral sclerosis (ALS), muscular dystrophy (MD), and stroke which in general, it leaves patients in a locked-in state [2]. Thus, Brain-Computer Interface (BCI) is introduced in 1970s by Vidal [3,4] providing a way to connect brain to the artificial or assistive device while bypassing the channel of peripheral nerves [2,5-7]. Three common types of $\mathrm{BCI}$ utilized in most BCI researches which are P300 Event-Related Potential, Steady-State Visual Evoke Potential (SSVEP), and Motor Imagery (MI) $[2,6,8]$. While $\mathrm{BCI}$ can be utilized by the user as a communication tool, signal processing plays a dominant role to validate the feasibility and reliability of the entire system. Two major parts in signal processing are feature extraction and classification algorithms in which the significant features are differentiated and segregated in accordance with which classes that they should be in prior to the measurement of the performance and classification accuracy [5].
Feature extraction technique on electroencephalography (EEG) signal has been popularly utilized in $\mathrm{BCI}$ as done by Pichiorri et al. [9] in conducting stroke patient rehabilitation. Apart from that, Fourier transform are utilized in extracting features which further being used in the BCI analysis as done by Hindarto et al. [10], Sivakami et al. [11], and Wang et al. [12]. The signal is often quantified based on their frequency characteristics and the spectrum is estimated using Fast Fourier Transform (FFT) [12]. However, FFT-based spectral analysis requires stationarity of the analyzed signal and it is well known that EEG is a non-stationary signal. Nevertheless, the time-varying spectrum of the signal can be obtained with time-frequency representation methods such as the traditional Short-Time Fourier Transform (STFT), Wavelet Transform (WT), and Autoregressive (AR) [14].

Apart from the feature extraction, classifier also plays a major role to produce robust BCI system which will be emphasized in this research. Nowadays, ubiquitous techniques for classification of feature had been tremendously applied in most BCI projects such as support 
vector machine (SVM) [15,16], K-nearest neighborhood (KNN) $[19,20]$, common spatial pattern (CSP) $[15,19]$, and linear discriminant analysis (LDA) $[20,21]$. However, most of the feature classification techniques are unstable when executing output of BCI signal. SVM is one of the most common method to isolate different classes of feature (for example, right and left class of hand movement) by constructing a hyperplane between those classes [20]. Nevertheless, SVM is unable to classify every single feature which might lead to the loss of important information in a particular feature. Thus, a robust technique should be designed in order to resolve the drawbacks encountered in the aforementioned researches.

Despite the growing BCI system development in modern countries, the development of BCI-based technology is still new in Malaysia and a lot of studies need to be carried out [21]. Since the BCI system requires a very robust decision-making signal processing, there are still many methods and techniques that may be explored to develop the most efficient BCI system which provides reliable and consistent outcomes.

In this paper, an algorithm for feature extraction integrated with classification in motor imagery experiment is proposed. The first objective is to design $\mathrm{BCI}$ signal processing to extract and segregate the most significant features from EEG signal to be utilized in BCI system by using fourth derivatives complex Gaussian of wavelet transform. The optimization procedure is employed on the extracted features on the stage of classification of features by implementing phase synchronization stability analysis technique with the adaptation of phase-locking measurement between two input signals. In addition, the algorithms are constructed to resolve the complexity issues thereby producing a stable and time-saving system. The second objective is to evaluate the performance of the proposed technique. It is expected that the proposed technique is able to produce high classification accuracy with a robust performance on motor imagery task. The phase stability approach has been successfully performed on classifying auditory selective attention [22]. Prior to that, Savitzky-Golay filter has been utilized to produce optimal signal-to-noise ratio (SNR) and is compared to the moving-average filter at the stage of signal preprocessing.

The main contribution of this work is that a new feature classification method was proposed combining with continuous wavelet transform as a feature extraction technique. The implementation of this technique may assist researchers in classifying multiclass features especially in the field of brain-computer interface research. Evaluation of performance accuracy provides guidance in deciding the technique to be utilized in any application.

\section{MATERIALS AND METHOD}

\section{Subject and data acquisition}

ten healthy subjects ( 5 males, 5 females) aged between 24 and 33 years old volunteered to participate in this experiment. Below is a set of paradigms that was used as the triggering stimulus for the subject in order to be align with BCI trials for this experiment. The experimental procedure took 2 minutes and during the experiment, it is necessary to avoid any noise interference which might affect the data acquisition. As shown in the Figure 1, the subject was asked to be ready at the prestimulus interval and stop the task at the post-stimulus.

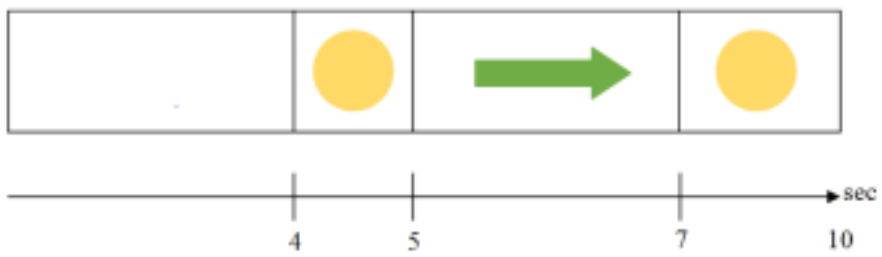

Figure $1 \mathrm{BCl}$ paradigm.

Prior to the motor imagery experiment, the subject was instructed to sit on a chair while facing towards the stimulus screen at the perpendicular position with the distance of approximately $100 \mathrm{~cm}$ (Figure 2). Throughout the procedure, the subject was required to wear the EEG scalp. Nuprep gel was used to improve skin condition for the electrodes attachment while reducing skin impedance. Next, a set of electrodes were attached to the scalp with the adhesion of Ten20 conductive gel as it has superior adhesive to hold non-disposable of neurodiagnostic electrode surface. Three active electrodes from GTEC's company were used over the sensorimotor areas, together with a reference electrode on the right ear lobe and ground electrode on the forehead. Three electrodes used were located on $\mathrm{C} 3, \mathrm{C} 4$, and $\mathrm{CZ}$, where most of motor planning and execution take place. The EEG signals were acquired using g.USBamp amplifier with $256 \mathrm{~Hz}$ sample frequency.

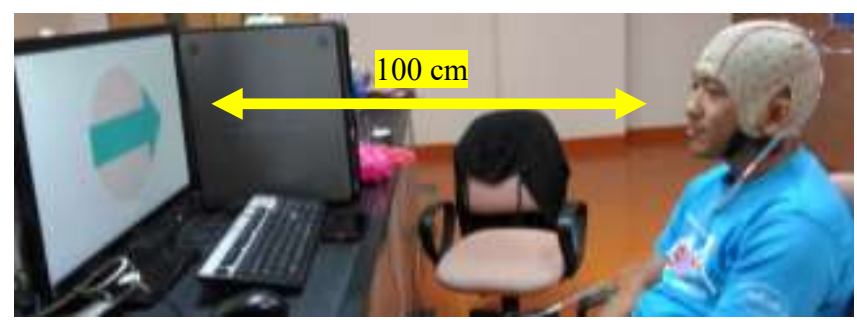

Figure 2 Subject on experiment.

\section{Signal preprocessing}

The EEG signals are dynamic with amplitude of $\pm 100 \mu \mathrm{V}$ without extended amplifier. However, it is usually contaminated by noise and artefact that might blur the important information. To solve this, Savitzky-Golay filter is used to denoise most of the noises underlying in EEG signals by applying linear least square method that is used to smooth the acquired EEG signals. This allows us to obtain high SNR while sustaining the original shape of the signals. The signal smoothing step applies equation 1 which was stated by Savitzky and Golay [23]. The equation consists of $n\left\{x_{j}, y_{j}\right\}$ points of $(j=1, \ldots, n)$.

$$
Y_{j=} \sum_{i=-\frac{m-1}{2}}^{\frac{m-1}{2}} C_{i} y_{j+i}
$$

where $x$ is an independent variable and $y$ is a dependent value. We applied the convolution set $m$ coefficient as the value of 3-point quadratic polynomial with the set of frame length value of $n=255$. The $j$ th values are in a range of

$$
\frac{m-1}{2} \leq j \leq n-\frac{m-1}{2}
$$

The performance of applied filter of signals were measured by the utilization of SNR technique. SNR is measurement used in engineering and science as to obtain the desired signal to the background noises. It can be defined as

$$
S N R=\frac{P_{\text {signal }}}{P_{\text {noise }}}
$$

where $P_{\text {signal }}$ is the power of the significant signal and $P_{\text {noise }}$ is the power of noise signal.

\section{Segmentation}

The significant filtered data were further processed by segmentation. In this process, the data were cut into ten folds which became ten trials (ten trials for right and ten trials for left-hand imagination). Similarly, this process was applied to another input which is real input but, in this phase, the input was recorded for only ten seconds in order to keep it as a "real time analysis". Different paradigm was utilized in this phase where the imagination of right- and left-hand were arbitrarily displayed on the screen with similar period per trial. 


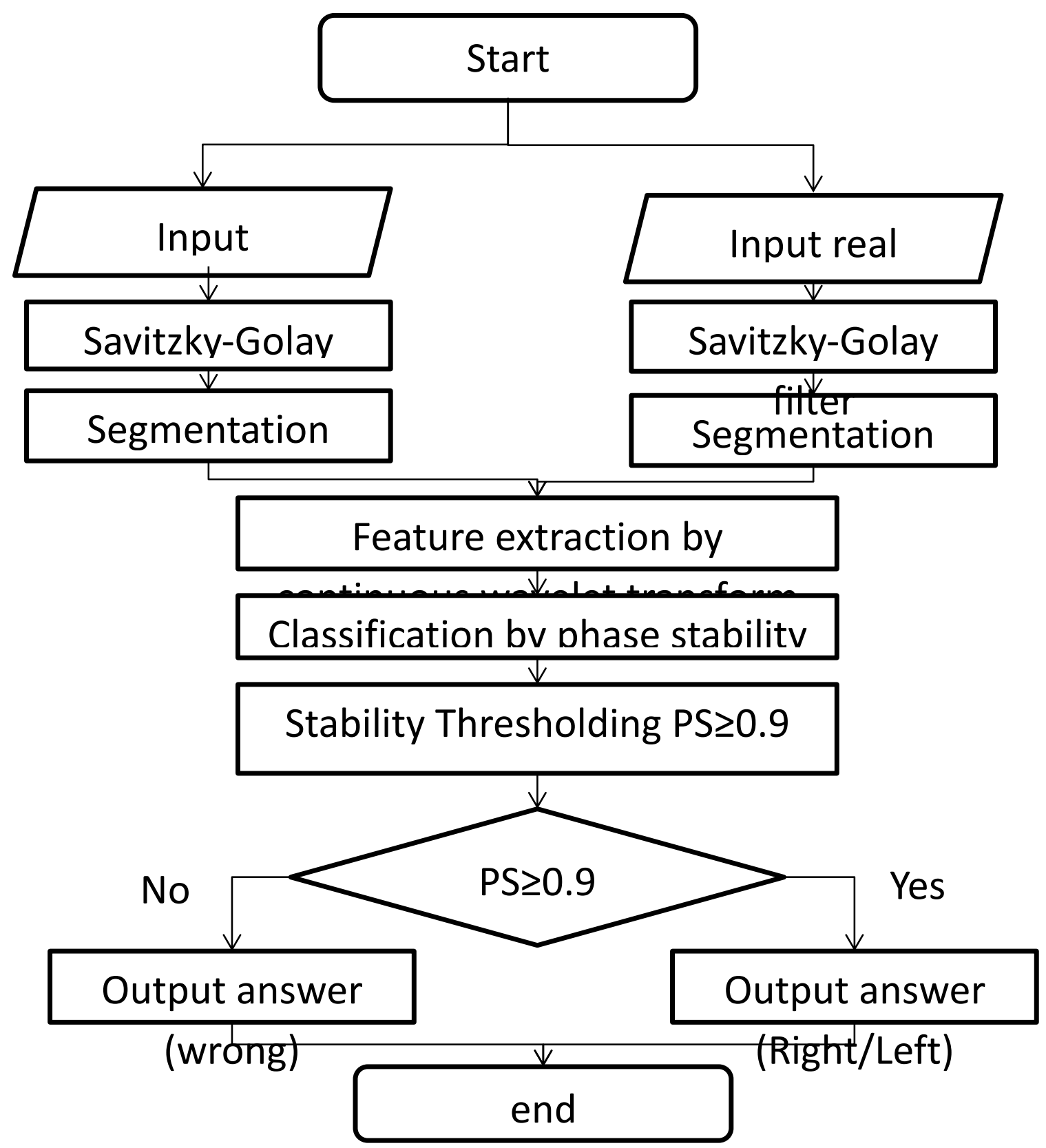

Figure 3 Flowchart of the methodology for algorithm.

\section{Feature extraction and classification technique}

Signals underwent the feature extraction process by continuous wavelet transform with complex Gaussian function of $4^{\text {th }}$ order derivative (based on equation (4)) where both reference and real inputs were compared. The scale parameter was applied by the value of 110 and pseudo frequency was obtained by

$$
f_{a}=T f_{\psi} / a
$$

where $f_{a}$ is denoted as frequency at instantaneous scale, $a$ is a scale, and $T f_{\psi}$ is a "pseudo" frequency at instantaneous scale.

As the phase stability analysis becomes the basis of signal processing used in our system, we considered the analysis of the synchronization stability, where the derived phase locking was adapted and implemented in the measured signals. Below is the mathematical expression for developing phase synchronization stability measurement.

In this study, $4^{\text {th }}$ derivative was utilized as a complex Gaussian function as wavelet by following the notations in Strauss et al. (2006)

$$
\psi_{a, b}(\cdot)=|a|^{-1 / 2} \psi((\cdot-b) / a)
$$

where $\psi \in L^{2}(\mathbb{R})$ is the wavelet with $0<\int_{\mathbb{R}}|\Psi(\omega)|^{2}|\Psi(\omega)|^{-1} \mathrm{~d} \omega<$ $\infty(\Psi(\omega)$ is the Fourier transform of the wavelet), and $a, b \in \mathbb{R}, a \neq 0)$. Thus, the wavelet transform is given as

$$
\mathcal{W}_{\psi}: L^{2}(\mathbb{R}) \rightarrow L^{2}\left(\mathbb{R}^{2}, \frac{\mathrm{d} a \mathrm{~d} b}{\mathrm{a}^{2}}\right)
$$

By following the notation from Rosenblum et al. (2001), the synchronization stability is defined as

$$
\Gamma_{a, b}(\mathcal{X}):=\frac{1}{M}\left|\sum_{m=1}^{M} e^{\imath \arg \left(\left(\mathcal{W}_{\psi} \chi_{m}\right)(a, b)\right)}\right|
$$


where $\Gamma_{a, b}$ of sequential $\chi=\left\{\chi_{m} \in L^{2}(\mathbb{R}): m=1, \ldots, M\right\}$ of $M$ sweeps. For the perfect neural synchronization, stability is indicated in a value of $(0,1)$. Although perfect coherent phases can be obtained, the stability tends to decrease as a result of phase jittering. Then, as the phase difference is obtained by

$$
e^{\imath\left(\arg \left(\left(\mathcal{W}_{\psi} \chi_{m}\right)(a, b)\right)-\arg \left(\left(\mathcal{W}_{\psi} y\right)(a, b)\right)\right)}
$$

where phase $\arg \left(\left(\mathcal{W}_{\psi} y\right)(a, b)\right)$ of a virtual reference signal $y$ in which $m$ is constant in scale and time. For this calculation, the result of stability remains unchanged. Although this is discernible in mathematical sense, it shows the relation of stability criteria to phase locking measurement form two signals and oscillators.

Extracted signals were measured in a similar technique, phase synchronization stability analysis where both reference and real time signals by the application of phase locking value (PLV) that varies from 0 to 1 . The higher the value indicated the better stability between two phases of input signals. Comparatively, this technique produces quite similar result as in the technique of wavelet-coherence and correlation coefficient. Nevertheless, it is observed that among these techniques, wavelet-phase stability is the most significant to be utilized in $\mathrm{BCI}$ system as it gives better significant differences between different classes of task.

In this process, the data was displayed in time-frequency representation and the level of stability was indicated by color-based image texture.

\section{Thresholding the phase stability features}

Thresholding is a method utilized in image processing to simplify the complexity of signal processing. Regardless, it also can be applied in this study as we used the time-frequency representation as the intensity-based segmentation. From the scale-time representation of classified signal, the selected data was summed from the value of averaged phase stability by the given condition

$$
P_{n, m}= \begin{cases}1 & p_{n, m} \geq T \\ 0 & p_{n, m}<T\end{cases}
$$

where $P_{n, m}$ is the resulting pixel of phase stability at the $(n, m)$ coordinate where $n$ is the $\mathrm{x}$-axis and $m$ is the $\mathrm{y}$-axis with the constant thresholding value, $T=0.9$. Thus, level of intensity-based representation that were less than the thresholding value were ignored while the coordinates more than thresholding value were subsequently summed up which consist of $\mathrm{C} 3, \mathrm{C} 4$, and $\mathrm{CZ}$ from reference and realtime experiment. Afterwards, it went through the process of decisionmaking followed the condition below. If:

$$
\begin{aligned}
& \text { 1. } P_{C 3}(\text { left })+P_{C 4}(\text { left })+P_{C Z}(\text { left })>P_{C 3}(\text { right })+ \\
& P_{C 4}(\text { right })+P_{C Z}(\text { right }) ; \text { then "left" output will be displayed. } \\
& \text { 2. } P_{C 3}(\text { right })+P_{C 4}(\text { right })+P_{C Z}(\text { right }) P_{C 3}>(\text { left })+ \\
& \quad P_{C 4}(\text { left })+P_{C Z}(\text { left }) ; \text { then "right" output will be displayed. } \\
& \text { 3. } P_{C 3}(\text { right })+P_{C 4}(\text { right })+P_{C Z}(\text { right }) P_{C 3}=(\text { left })+ \\
& \quad P_{C 4}(\text { left })+P_{C Z}(\text { left }) ; \text { output will display "try again". }
\end{aligned}
$$

The evaluation of BCI system performance was constructed based on the method of confusion matrix [24]. This method is defined as performance accuracy of classification between two classes i.e. righthand imagination and left-hand imagination. The advantage of this performance evaluation is the practice of examining large database can be easily observed to predict if the designed model is confusing the matrix, for instance, mislabeling as one another. Besides, it shows the percentages of correctly classified data over the total number of data inputs into the confusion matrix. Furthermore, the overall average accuracy would also be quantified.

The confusion matrix model was designed based on the total outcomes of the ten participants. The model constitutes for types of predictive variables which are true positives (TP), true negatives (TN), false positives (FP), and false negatives (FN). Below are the descriptions for every term from the designed model:

1. True positives (TP): These are cases when we predicted 'yes' for they are able to produce correct outcomes (which in this experiment, right or left-hand imagination outcome).

2. True negatives (TN): We predicted "no", and they produce wrong outcomes.

3. False positives (FP): We predicted "yes", but they actually produce wrong outcomes.

4. False negatives (FN): We predicted "no", but they produce correct outcomes.

\section{RESULTS AND DISCUSSION}

\section{Experimental results}

In the present study, it was observed that the acquired EEG signals based on selected electrodes which are $\mathrm{C} 3, \mathrm{C} 4$, and $\mathrm{CZ}$ displayed nonerror oscillations. The raw signals were then undergoing the segmentation process where the raw signals were cut into 10 trials and each trial contains 2560 samples. In Figure 4.4, it shows a plotted graph of amplitude against time in seconds for single trial. Each of the segmented signal was smoothed by using Savitzky-Golay filter. The filter was compared with another signal smoothing method namely moving-average (MA) filter in order to choose better implementing filter.

Signals comprise of both desirable and undesirable information that might contain a frequency or a range of frequencies. Moving average filter is a low-pass filter (LPF) which averaging all data points. MA filter is a type of filter that easy to understand, thus it is applied in most research. In the table, it shows the filtered signal by SavitzkyGolay filter, where the output is almost identical to the output signal by MA filter. However, it was clearly shown that there exist major differences of amplitude size between the signals. 


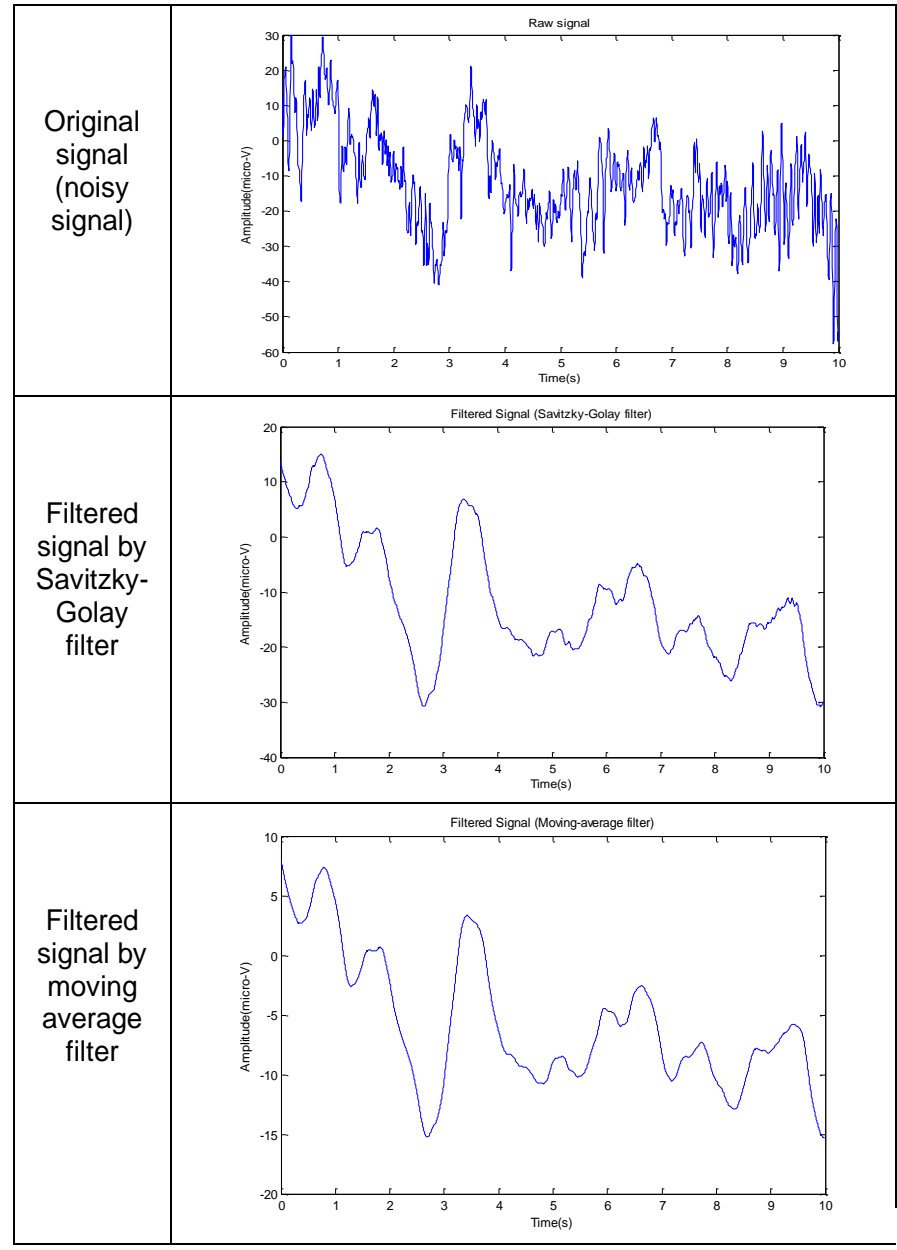

Table 2 Comparison between original and filtered signals.

\begin{tabular}{|c|c|c|}
\hline $\begin{array}{c}\text { Frame/window } \\
\text { length }\end{array}$ & S-G filter (SNR) & M-A filter (SNR) \\
\hline 255 & 6.2914 & 2.0049 \\
\hline 127 & 8.3706 & 3.6403 \\
\hline 63 & 9.5778 & 5.3146 \\
\hline
\end{tabular}

From Table 2, it shows the comparison between two filters that had been discussed. The value of window and frame length are varied and a large gap of SNR values can be observed between both filters. This shows that the implementation of Savitzky-Golay filter outperforms the MA method. Similar previous research on EEG experiment also implemented these techniques. The results reaffirm that Savitzky-Golay method produces better SNR than MA filter.

\section{Analysis of decision outcomes}

Analyses conducted were focusing on two outcomes which are right-hand decision and left-hand decision. Table 3 shows the comparison for left and right imagination tasks in the form of scale against time representation of wavelet-phase stability analysis. The analysis of stability was measured between two inputs signal which are reference signal and real-time signal. The stability between these two signals were assessed by indicating a range of phase stability from 0 which is unstable to 1 which is the highest stability in this evaluation. High intensity of stability values was dominated on the first second until the third second for both right and left imagination. It was observed that the right imagination (targeted output) produced higher intensity for every channel which are $\mathrm{C} 3, \mathrm{C} 4$, and $\mathrm{CZ}$ than left imagination (non-targeted output). It implies that the subject was able to trigger right-hand output when the online motor imagery task was in progress while rejected the left imagination analysis as it produced lower intensity.
Table 3 Right-hand decision outcome.

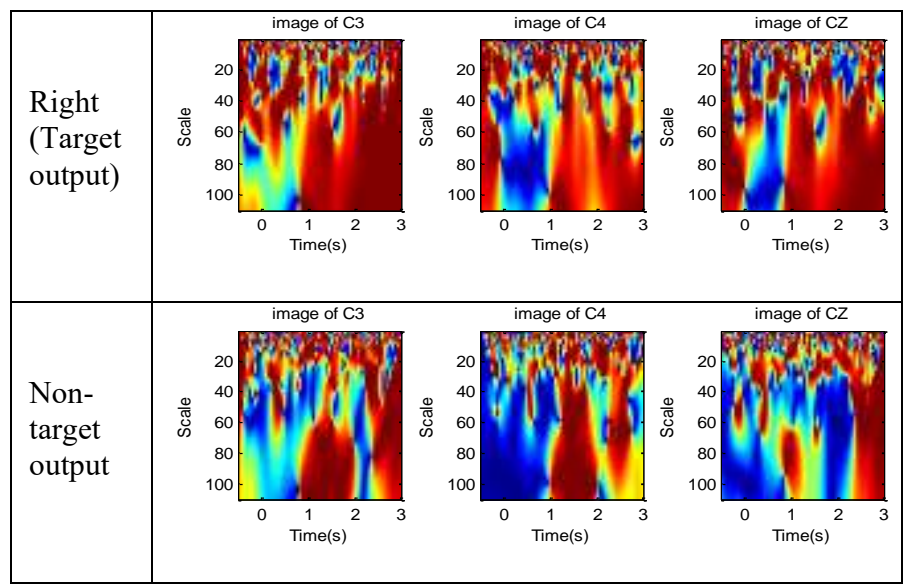

Table 3.1 The general calculation of confusion matrices. This technique is widely used in evaluating the performance of classification model.

\begin{tabular}{|c|c|c|c|c|}
\hline \multirow{4}{*}{ Predicted } & \multicolumn{3}{|c|}{ Actual } \\
\hline & & Target & $\begin{array}{c}\text { Non- } \\
\text { target }\end{array}$ & Total \\
\cline { 2 - 6 } & Target & TP & FP & TP+FP \\
\cline { 2 - 6 } & $\begin{array}{c}\text { Non- } \\
\text { target }\end{array}$ & FN & TN & FN+TN \\
\hline \multicolumn{2}{|c|}{ Total } & TP+FN & FP+TN & TP+FP+FN+TN \\
\hline
\end{tabular}

In contrast, motor imagery experiment was also conducted to observe the left motor output. But it can be seen that the domination of high intensity was produced throughout the time for both right and left imagination on all channels. However, it shows that intensity was produced higher on the left imagination (targeted output) than the right imagination (non-targeted output) of reference and real time signals measurement. Thus, results on Table 4 shows the subject for this task was successfully performs left-hand imagination of decision output.

Table 4 Left-hand decision outcome.

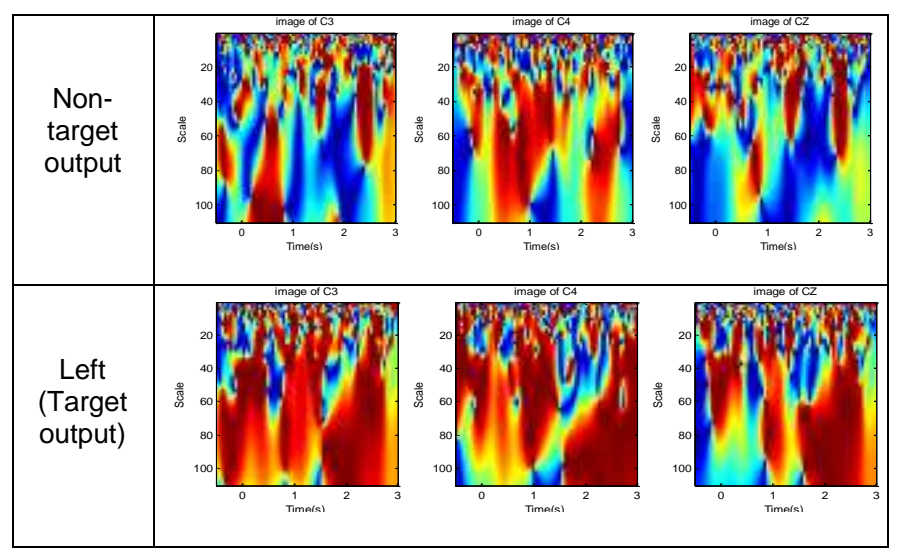

Next, Figure 4 shows the bar graph of total presence of true imaginary for right-hand imagination and left-hand imagination which are indicated in blue and orange bars, respectively. On the y-axis, the total of present imagination refers to a situation where subjects were able to produce correct decision outcomes based on the tasks given. From the graph, it can be seen that the highest number of total right imaginary outcome is on subject 2 with a score 10 out of 10 while the total number of lowest was on subject 9 with 6 scores. On the other 
hand, for the left-hand imaginary, subject 2 was able to produce 8 true outcomes while the lowest hit on subject 5 with 6 scores. Overall, the total imaginary of right decision outcomes were higher than the left outcomes. We can conclude that most of the participants tend to perform better on right-hand imaginary than the other side. However, this result could still be improved and balanced as to produce a firm and robust BCI signal processing technique.

\section{Total imaginary}

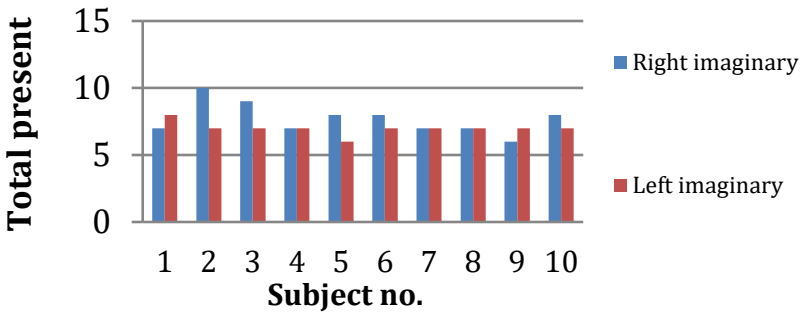

Figure 4 Flowchart of the methodology for algorithm.

\section{Performance evaluation}

The evaluation of the implemented BCI processing was observed by using the technique of confusion matrices. Two types of measurement that had been utilized in this method are the calculation of confusion matrix by right imagination as the output decision (see Table 4.5) and left imagination as the output decision (see Table 4.6) Finally, the confusion matrices were constructed with the determination of false positives, false negatives, true positives, and true negatives based on all participants results.

There were 200 numbers of outcomes evaluated on confusion matrices technique where 100 for left decision outcomes and another 100 from right decision outcomes. From Table 5, it shows that all participants produced 107 of total predicted outcomes and 93 outcomes for non-target outcomes. As the validation of performance, it shows the model was successful in attaining $72 \%$ of overall operation of the system which is considered as a good performance. Besides, based on the classification algorithm, this model was able to classify $75 \%$ precision of overall proportion in producing targeted outputs which correspond with the actual output decisions. Furthermore, this model also produced $77 \%$ sensitivity that tells us the proportion of actual target outcome, which in this case is right outcomes was predicted by algorithm as producing targeted outcomes. Next, for the specificity, $70 \%$ of the proportion of participants did not produce non-targeted outcomes and were predicted by the model as non-targeted (which is in this case is left outcomes).

Table 5 Confusion matrix for right-hand decision as a target output.

\begin{tabular}{|c|c|c|c|c|}
\hline & \multicolumn{4}{|c|}{ Actual } \\
\hline \multirow{3}{*}{ Predicted } & Right & Right & Non-right & Total \\
\cline { 2 - 5 } & $\begin{array}{c}\text { Non- } \\
\text { right }\end{array}$ & 23 & 30 & 107 \\
\hline \multicolumn{2}{|c|}{ Total } & 100 & 70 & 93 \\
\hline
\end{tabular}

From Table 6, it shows that all participants produced 93 of total predicted outcomes which is left-hand and 107 outcomes for non-target outcomes which is right hand. As the validation of performance, it shows the model achieved $74 \%$ of overall operation of the system which is considered as good performance as similar as the preceded confusion matrices. Furthermore, from the classification algorithm, this model was able to classify $75 \%$ precision of overall proportion in producing targeted outputs correspond with the actual output decisions. On the other hand, this model produced $70 \%$ sensitivity that tells us the proportion of actual target outcome, which in this case is left outcomes was predicted by algorithm as producing targeted outcomes. Next, $77 \%$ specificity was reported where $77 \%$ of the participants did not produce non-targeted outcomes and were predicted by the model as nontargeted (which is in this case is right outcomes).
Table 6 Confusion matrix for left-hand decision as a target output.

\begin{tabular}{|c|c|c|c|c|}
\hline & \multicolumn{4}{|c|}{ Actual } \\
\hline \multirow{3}{*}{ Predicted } & & Left & Non-left & Total \\
\cline { 2 - 5 } & Left & 70 & 23 & 93 \\
\cline { 2 - 5 } & Non-left & 30 & 77 & 107 \\
\hline \multicolumn{2}{|c|}{ Total } & 100 & 100 & 200 \\
\hline
\end{tabular}

From the evaluation of the performance, it shows that the implemented classification algorithm was able to attain high accuracy of more than $70 \%$ which is the benchmark of good performance. From both calculation of confusion matrices tables, it shows a balanced trade off of the result thereby affirmed the model as considerable and significant.

The results were compared with the previous studies to obtain the benchmarks for the classification accuracy. Previously, a research was conducted on implementing the support vector machine (SVM) as a classification of features and result produced below $70 \%$ of classification accuracy [13]. Although SVM is one of the most common methods used to isolate different classes of feature, it is unable to classify every single feature which might lead to the loss of important information in particular features. Apart from that, in [25], BCI experiment was conducted by using K-Nearest Neighbor approach as a classification of feature. With the average accuracy of 53\%, this shows that K-Nearest Neighbor approach has low reliability in solving multiclass problems. These imply that our proposed technique produced better results and can be utilized in classifying multiclass problems.

Nevertheless, there are other methods that are far superior than the proposed technique in solving problems of other related disciplines. For instance, a technique utilized by [26] produced $81 \%$ of accuracy using Linear Discriminant Analysis (LDA). The advantage of utilizing that technique is that LDA is mathematically simple and computationally efficient but additional feedback delay may occur during computational process. For our technique, it is feasible to measure large neural correlates. Thus, it produces more meaningful data while acquiring the broad-band signal phase.

\section{CONCLUSION}

In this paper, we proposed the algorithm based on phase stability analysis for feature classification of two-class motor imagery-based brain-computer interface. The new method was incorporated with continuous wavelet transform as a feature extraction. Prior to that, a signal smoothing technique was implemented which is Savitzky-Golay filter to optimize the signal-to-noise ratio (SNR). Experimental results have shown that with overall classification accuracy over $70 \%$, it can be concluded that the proposed technique is considerably good and reliably utilized. Future works may involve other types of BCI experiment such as P300 event-related potential and steady-state visual evoke potential for further application of phase stability analysis.

\section{ACKNOWLEDGEMENT}

The authors gratefully acknowledge the research grant provided by Research Management Centre which was sponsored by Ministry of Education, Malaysia, Q.J130000.3051.01M61 (Research University Grant), Universiti Teknologi Malaysia, Johor Bahru, Malaysia. 


\section{REFERENCES}

[1] Zulkiply, R. Registration of PWD's by Category of Disabilities. Report on Statistic of Department of Welfare Society. (2014). 167.

[2] Wolpaw, J. R., Birbaumer, N., McFarland, D. J, Pfurtscheller, G., Vaughan, T. M. Brain-computer interfaces for communication and control. Clinical Neurophysiology. 2002. 113(6), 767-791.

[3] Vidal, J. J. Toward direct brain-computer communication. Annual Review of Biophysics and Bioengineering. 1973. 2(1), 157-180.

[4] Vidal, J. J. Real-time detection of brain events in EEG. IEEE Proceedings. 1977. 65(5), 633-641.

[5] Al-Ani, Tarik, Trad, D. Signal Processing and Classification Approaches for Brain-computer Interface, Intelligent and Biosensors, InTech, 2010, 25-66.

[6] G. Bernhard, B. Allison, G. Pfurtscheller, Brain-computer Interfaces: A Gentle Introduction, In G. Bernhard, B. Allison, Pfurtscheller, eds. Brain-Computer Interfaces, Berlin: Springer, 2009, 1-27.

[7] Abdulkader, Sarah, N., Atia, A., Mostafa-Sami M. Mostafa, M.-S. M Brain computer interfacing: Applications and challenges. Egyptian Informatics Journal. 2015. 16(2), 213-230.

[8] Lazarou, I., Nikolopoulos, S., Petrantonakis, P. C., Kompatsiaris, I., Magda Tsolaki, M. EEG-based brain computer interfaces for communication and rehabilitation of people with motor impairment: A novel approach of the 21 st century. Frontiers in Human Neuroscience. 2018. 12, 14

[9] Pichiorri, M., Morone, G., Petti, M., Toppi, J., Pisotta, I., Molinari, M. Paolucci, S., Inghilleri, M., Astolfi, L., Cincotti, F., Mattia, D. Braincomputer interface boosts motor imagery practice during stroke recovery. Annals of Neurology. 2015. 77(5), 851-865.

[10] Hindarto, H., Sumarno S. Feature extraction of electroencephalography signals using fast fourier transform. CommIT (Communication and Information Technology) Journal. 2016. 10(2), 49-52.

[11] Sivakami, A., S. Shenbaga Devi. Analysis of EEG for motor imagerybased classification of hand activities. International Journal of Biomedical Engineering and Science. 2015. 2(3), 11-22.

[12] Jie, W., Feng, Z., Lu, N. Feature extraction by common spatial pattern in frequency domain for motor imagery tasks classification. $29^{\text {th }}$ Control and Decision Conference (CCDC). 2017. Chinese: IEEE.

[13] Begic, D., Mahnik-Milos, M., Grubisin, J. EEG characteristics in depression, "negative" and "positive" schizophrenia. Psychiatr Danub. 2009, 579-584.

[14] Ubeyli, E. D., Cvetkovic, D., Cosic, I., AR spectral analysis technique for human PPG, ECG and EEG signals. Journal of Medical Systems. 2008. 32(3), 201-206.

[15] Mondini, Valer ia, Anna Lisa Mangia, and Angelo Cappello. "EEGBased BCI system using adaptive features extraction and classification procedures." Computational intelligence and neuroscience 2016 (2016).

[16] Rathipriya, N., S. Deepajothi, and T. Rajendran. "Classification of motor imagery ecog signals using support vector machine for brain compute interface." Advanced Computing (ICoAC), 2013 Fifth International Conference on. IEEE, 2013.
[17] Imran, S. M., Talukdar, M. T. F., Sakib, S. K., Pathan, N. S., Fattah, S. A. Motor imagery EEG signal classification scheme based on wavelet do main statistical features. Electrical Engineering and Information \& Communication Technology (ICEEICT), 2014 International Conference. 2014. 10-12 April, Dhaka: IEEE.

[18] Bashar, Khairul, S., Hassan, A. R., Bhuiyan, M. I. H. Identification of motor imagery movements from EEG signals using dual tree complex wavelet transform. Advances in Computing, Communications and Informatics (ICACCI), 2015 International Conference. 2015. 10-13 August, India: IEEE.

[19] Ortner, R., Scharinger, J., Lechner, A., Guger, C. How many people can control a motor imagery based BCI using common spatial patterns? Neural Engineering (NER), 2015 7th International IEEE/EMBS Conference. 2015. 22-24 April, France: IEEE.

[20] Nicolas-Alonso L. F., Corralejo, R., Gomez-Pilar, J., Álvarez, D., Hornero R. Adaptive stacked generalization for multiclass motor imagery-based brain computer interfaces. IEEE Transactions on Neural Systems and Rehabilitation Engineering. 2015. 23(4), 702-712.

[21] Guger, C., Spataro, R., Allison, B. Z., Heilinger, A., Ortner, R., Cho, W., La Bella, V. Complete locked-in and locked-in patients: command following assessment and communication with vibro-tactile P300 and motor imagery brain-computer interface tools. Frontiers in Neuroscience.2017. 11, 251.

[22] Singla, Shubham, S. N. Garsha, Chatterjee, S. Characterization of classifier performance on left and right limb motor imagery using support vector machine classification of EEG signal for left and right limb movement. Wireless Networks and Embedded Systems (WECON), 2016 5th International Conference. 2016. 14-16 October, India: IEEE.

[23] Ooh, A. A., Yunus, J., Daud, S. M. A review of asynchronous electroencephalogram-based brain computer interface systems. International Conference on Biomedical Engineering and Technology IPCBEE. 2011.

[24] Low, Fen, Y., Strauss, D. J. A performance study of the wavelet-phase stability (wps) in auditory selective attention. Brain Research Bulletin. 2011. 86(1-2), 110-117.

[25] Savitzky, A. Golay, M. J. Smoothing and differentiation of data by simplified least squares procedures. Analytical Chemistry. 1964. 36(8), 1627-1639.

[26] Markham, K., Simple Guide to Confusion Matrix Terminology. Data school. 2014

[27] Liu, C., Wang, H., Lu, Z. EEG classification for multiclass motor imagery BCI. Control and Decision Conference (CCDC), 2013 25th Chinese. 2013. 25-27 May, China: IEEE.

[28] Bashar, S. K., Hassan, A. R., Bhuiyan, M. I. H. Identification of motor imagery movements from EEG signals using dual tree complex wavelet transform. Advance in Computing Communications and Informatics (ICACCI), 2015 international Conference on. 2015. 10-13 August, India: IEEE. 